\title{
LE OPINIONI DEGLI INSEGNANTI SULL'INSEGNAMENTO IN CLASSI LINGUISTICAMENTE E CULTURALMENTE
}

\section{ETEROGENEE}

\author{
Anja Zorman, Univerza na Primorskem, Università del Litorale, \\ anja.zorman@fhs.upr.si
}

10.31902/fll.30.2020.22

UDK 811.131.1:811.162.4]:371.3

\begin{abstract}
Nel presente contributo vengono esposti alcuni risultati della ricerca sull'interculturalità e sull'educazione interculturale, condotta in sei regioni slovene e italiane con presenza di minoranze linguistiche e culturali. La ricerca ha preso in considerazione vari aspetti della didattica, della formazione degli insegnanti, degli atteggiamenti degli allievi e dei loro genitori riguardo all'apprendimento in classi linguisticamente e culturalmente eterogenee. I risultati presentati nel contributo riguardano le opinioni degli insegnanti sull'insegnamento in classi linguisticamente e culturalmente eterogenee, suddivise in seguenti categorie: (1) lo sviluppo professionale, (2) personale e culturale degli insegnanti coinvolti nelle classi linguisticamente e culturalmente eterogenee, (3) la loro percezione dell'apprendimento in generale e linguistico in particolare, e (4) delle relazioni interpersonali tra gli allievi. Dallinchiesta che ha coinvolto 281 insegnanti emerge una percezione degli insegnanti molto positiva del loro sviluppo professionale e culturale, e neutra riguardo la collaborazione con altri insegnanti. Sebbene la maggioranza degli insegnanti non sia dell'opinione che la compresenza di allievi di diverse origini linguistiche e culturali ostacoli il lavoro in classe, dichiara comunque che l'insegnamento in classi linguisticamente e culturalmente eterogenee richiede lavoro aggiuntivo. La didattica non risente delle difficoltà nella comunicazione tra allievi di lingue e culture diverse, così come lo sviluppo della competenza comunicativa della lingua d'insegnamento non è compromesso dalla eterogeneità linguistica in classe. Molto positiva è la percezione degli insegnanti relativa allo sviluppo di rapporti interpersonali tra gli allievi.
\end{abstract}

Parole chiave: classi linguisticamente e culturalmente eterogenee, opinioni degli insegnanti, didattica, educazione interculturale, italiano, sloveno 


\section{Introduzione}

Le percezioni che gli insegnanti hanno del proprio lavoro e dell'informazione, diretta o indiretta, che su di esso ricevono dall'ambiente ${ }^{1}$ sono strettamente legate alla qualità della loro didattica e della loro vita professionale in generale. Gli insegnanti che sono in sintonia con la cultura organizzativa della scuola sono meno soggetti allo stress professionale. Ciò significa che nelle scuole con forte presenza di allievi di diversa provenienza linguistico-culturale gli insegnanti dotati della sensibilità interculturale lavoreranno meglio e saranno meno estenuati fisicamente, mentalmente ed emotivamente se la cultura organizzativa generale della scuola si basa sul rispetto e sull'empatia verso il diverso (Tatar, Horenczyk 405). Non solo, dalla ricerca di Pederson (18) emerge che l'educazione interculturale a livelli più alti di sensibilità interculturale (Bennett 14$)^{2}$ si può sviluppare solo in ambienti scolastici che permeano completamente la didattica e la vita della scuola in generale, compreso tutto il personale, non solo gli insegnanti. Dal confronto di tre scuole coinvolte nella ricerca di Pederson (18) è apparso che gli allievi della scuola situata nell'ambiente urbano mostravano atteggiamenti meno tolleranti verso la diversità culturale rispetto ai loro coetanei della scuola in periferia, e che le differenze tra i due gruppi erano statisticamente significative. Una scuola con un numero elevato di allievi di background linguisticoculturale diversi non può ignorare la loro presenza, intesa come un gruppo visibile con particolari necessità psicologiche e didattiche (Tatar, Horenczyk 404), tuttavia il contatto con la diversità, senza dubbio uno dei fattori essenziali allo sviluppo della sensibilità

${ }^{1}$ Qui ci si riferisce al comportamento degli allievi in classe, alla loro motivazione per lo studio e al loro rendimento scolastico, inoltre alle pressioni dall'esterno, sia dalla scuola che dai genitori e dalla società in generale, alla retribuzione che considerano inadeguata all'impegno investito nel lavoro, e alle insufficienti opportunità per lo sviluppo personale (Farber 592).

${ }^{2} \mathrm{Nel}$ proprio modello dello sviluppo della sensibilità interculturale Bennett (19-25) distingue principalmente tra livelli etnocentrici e livelli etnorelativi. Si considerano livelli più alti, quelli che si dovrebbero possedere per funzionare con successo negli ambienti plurilingui e pluriculturali, i livelli etnorelativi: (a) l'accettazione, basata sul rispetto delle differenze comportamentali e dei valori; (b) l'adattamento che si può verificare a patto che la persona possieda o sviluppi l'empatia verso l'altro, e il pluralismo, inteso come la capacità della persona di fare affidamento su diversi schemi di riferimento o schemi culturali multipli; e (c) I'integrazione che richiede una valutazione costruttiva e una definizione continua dell'identità del singolo individuo in termini di esperienze vissute. 
interculturale a livelli più alti, non è necessariamente bastante (Zudič Antonič 612). L'ethos scolastico e la sua cultura organizzativa generale, che deriva sia dal curricolo scritto che quello nascosto, hanno fondamentale importanza nello sviluppo della sensibilità interculturale degli allievi e della percezione positiva del proprio lavoro da parte degli insegnanti.

Una positiva percezione di sé come figura professionale, che deriva da una parte da una preparazione adeguata per il lavoro in classi eterogenee, e da una naturale disposizione personale ad agire in tali contesti, e dall'altra parte dal senso di realizzazione professionale e personale contribuisce alla motivazione per il lavoro in classe, per l'aggiornamento continuo in vari aspetti legati alla professione dell'insegnante, per lo scambio di esperienze con altri insegnanti, per la partecipazione ai progetti di ricerca. Gli insegnanti che invece sentono il proprio lavoro come stressante forniscono significativamente meno informazioni agli allievi, li lodano meno, si dimostrano meno disponibili ad accettare le idee degli allievi e comunicano in generale meno con loro.

La sensibilità interculturale è una capacità importante degli insegnanti che lavorano con successo in classi demograficamente eterogenee (Zorman, Zudič Antonič 254). L'insegnante ha un forte impatto sul rendimento scolastico degli allievi, in particolare di quelli che provengono da famiglie socialmente più deboli e da famiglie di diversi retroterra. Ciò nonostante le ricerche mostrano che gli insegnanti hanno minori aspettative e interagiscono meno proprio con questi allievi (Tucker et al. 29), che di conseguenza diventano spesso allievi a rischio di fallimento scolastico.

Le precedenti ricerche tra studenti (pre-service) e insegnanti (in-service) si sono occupate prevalentemente con la loro preparazione per l'insegnamento in classi eterogenee (Ladson-Billings 1999; Dong 2004; Sleeter 2008; Lehman 2017) ${ }^{3}$ in contesti con una scarsa presenza della minoranza nel corpo docente (Sleeter 2001). A tal proposito è nato un filone di ricerca sui valori e sulle convinzioni dei (futuri) insegnanti con lo scopo di individuare i fattori chiave che conducono allo sviluppo della sensibilità interculturale (Garmon 2004; Pohan 2006), sviluppare corsi di formazione, modalità di valutazione e sistemi organizzativi della classe e della scuola in generale (Tatto 1996) e sviluppare strumenti di valutazione dei corsi di formazione (Walker 2008). Un altro importante filone di ricerca si occupa dello sviluppo

\footnotetext{
${ }^{3}$ Nel presente capoverso, dato il riferimento al testo integrale viene citato, insieme all'autore, l'anno della pubblicazione.
} 
della competenza interculturale nel corso della formazione dei futuri insegnanti (Moon, Callahan, Tomlinson 1999; McAllister 2000) e come parte dello sviluppo professionale degli insegnanti già operanti nel sistema scolastico (Knight, Wiesman 2009), realizzato anche attraverso modelli di tutoraggio tra pari (Galbraith, Anstrom 1995). Le ricerche che si sono dedicate alle opinioni sia degli insegnanti in corso di formazione che insegnanti in servizio miravano soprattutto alle opinioni sulla diversità (Tatto 1996; Pohan 2006; Walker 2008). La nostra ricerca si è impegnata invece a scoprire in quale modo e in quale misura l'insegnamento in classi eterogenee sia influenzato, secondo la loro opinione, dalla presenza di allievi di diversa origine linguistico-culturale. $\mathrm{Ci}$ siamo interessati a quegli aspetti dell'insegnamento in classi eterogenee che nei contatti precedenti, durante corsi di formazione, conferenze e in altre occasioni, gli insegnanti hanno esposto come problematici dal punto di vista dello svolgimento del processo didattico: I'andamento soprattutto in termini di dinamica, quantità del carico di lavoro dell'insegnante, il livello di competenza della lingua d'insegnamento della scuola e problemi di comunicazione tra gli allievi. Partendo dal presupposto che il lavoro degli insegnanti sia esigente e può essere fonte di forte stress fisico, psicologico e mentale, il processo didattico non ne risentirà qualora gli insegnanti abbiano una percezione positiva della propria figura professionale e possiedano alti livelli di sensibilità interculturale. In base ai dati raccolti sul campo si è cercato di dedurre in quale relazione siano le percezioni degli insegnanti e la loro sensibilità interculturale.

\section{Metodologia}

La ricerca di cui vengono presentati alcuni risultati in questo articolo è stata condotta come parte del progetto EDUKA - Educare alla diversità (2011-2014) - in cui sono state coinvolte sei istituzioni partner in Slovenia e in Italia. ${ }^{4}$ Per la ricerca sono state selezionate scuole con alta presenza di diversità linguistica, culturale ed etnica in

${ }^{4}$ Centro di Ricerca sloveno SLORI (Trieste, Italia), Università del Litorale, Facoltà di Studi Umanistici (Capodistria, Slovenia), Università di Trieste, Dipartimento di Scienze Politiche e Sociali (Trieste, Italia), Università di Udine, Dipartimento di Antropologia (Udine, Italia), Università Ca' Foscari di Venezia, Dipartimento di Scienze Linguistiche e Culturali Comparative (Venezia, Italia), Centro di Ricerca dell'Accademia di Scienze e Arti di Slovenia, Istituto sulle Migrazioni (Lubiana, Slovenia). 
sei regioni dei due stati partecipanti. ${ }^{5}$ I dati sono stati raccolti per mezzo del questionario, distribuito agli allievi di età dai 12 ai 15 anni, ai loro genitori e insegnanti. Nel presente contributo vengono presentati e discussi alcuni risultati emersi dalla ricerca.

Agli insegnanti è stato chiesto di esprimere le proprie opinioni sull'insegnamento in classi linguisticamente e culturalmente eterogenee, in particolare sul loro sviluppo professionale, personale e culturale, sulla loro percezione dell'apprendimento in generale e linguistico in particolare in classi con presenza di allievi di diverso background linguistico-cultuale, e sulle relazioni interpersonali tra gli allievi in tali classi.

\section{Campione}

Alla ricerca hanno preso parte 281 insegnanti che nell'anno scolastico 2012/13 hanno lavorato con allievi di età dai 12 ai 15 anni in sei regioni slovene e italiane. $77,9 \%$ erano femmine e $21,4 \%$ maschi, $^{6}$ con un'età media di 44,84 anni. $47.0 \%$ erano insegnanti di lettere, $23,1 \%$ insegnanti di scienze, seguiti da insegnanti di arte $(11.0 \%)$, di educazione fisica (5,3\%), di attività extracurriculari $(5,0 \%)$ di tecnica $(4,3 \%){ }^{7} 51,2 \%$ di insegnanti ha insegnato una sola materia, $22,8 \%$ ne ha insegnate due, $12,8 \%$ tre, $6,4 \%$ quattro, $1,4 \%$ cinque e $1,1 \%$ sei materie. ${ }^{8}$ La media di esperienza didattica degli insegnanti interpellati era 17.68 anni.

\section{Risultati e discussione}

I dati sulle opinioni degli insegnanti sono stati raccolti con la scala Likert, contenente alcune affermazioni sull'insegnamento nelle classi eterogenee, ciascuna accompagnata da una gamma di possibili giudizi offerti alla scelta della persona interpellata. Alle prime sette affermazioni discusse nel presente contributo sono stati associati i seguenti giudizi: 1 - per niente d'accordo, 2 - non d'accordo, 3 indeciso, 4 - d'accordo e 5 - completamente d'accordo. All'ultima

\footnotetext{
${ }^{5}$ Nelle aree bilingui sul Litorale Sloveno e della regione Friuli-Venezia Giulia in Italia hanno partecipato scuole con la lingua d'insegnamento slovena (maggioritaria in Slovenia minoritaria in Italia) e scuole con la lingua d'insegnamento italiana (maggioritaria in Italia e minoritaria in Slovenia); nella parte centrale dei due paesi hanno partecipato scuole in Lubiana (Slovenia), Venezia e Ravenna (Italia).

${ }^{6} 0.7 \%$ di insegnanti non ha risposto a questa domanda.

${ }^{7} 4,3 \%$ di insegnanti non ha risposto a questa domanda.

${ }^{8} 4,3 \%$ di insegnanti non ha risposto a questa domanda.
} 
affermazione, relativa all'influenza della presenza di allievi di diversa provenienza linguistico-culturale in classe, i giudizi associati sono stati: 1 - molto negativo, 2 - negativo, 3 - né negativo né positivo, 4 - positivo e 5 - molto positivo.

I risultati emersi dalla ricerca sono presentati in tabelle, seguite dall'interpretazione e discussione dei dati che riportano. La colonna a sinistra contiene i giudizi offerti agli insegnanti dal questionario, le colonne centrali riportano le percentuali delle risposte per ciascuna delle sei regioni coinvolte nella ricerca e la colonna a destra contiene la media delle risposte dell'intero campione. I risultati discussi sono suddivisi in seguenti categorie: (1) lo sviluppo professionale, (2) personale e culturale degli insegnanti coinvolti nelle classi linguisticamente e culturalmente eterogenee, (3) la loro percezione dell'apprendimento in generale e linguistico in particolare, e (4) delle relazioni interpersonali tra gli allievi nelle classi in cui insegnano.

Lo sviluppo professionale dell'insegnante. Agli insegnanti è stato chiesto se il lavoro nelle classi eterogenee li stimola a partecipare a un continuo aggiornamento professionale. La maggior parte degli insegnanti concorda con l'affermazione. Le percentuali 33,3\% e 33,1\% nella colonna 'Totale' (Tabella 1) indicano che un terzo degli insegnanti si dichiara completamente d'accordo e un terzo si dichiara d'accordo con l'affermazione. Le altre percentuali nella stessa colonna dimostrano che $23,8 \%$ degli insegnanti è indeciso e che solo una minima parte è dell'opinione che il lavoro nelle classi eterogenee non stimoli $(6,4 \%)$ ovvero non stimoli affatto $(2,8 \%)$ a partecipare all'aggiornamento professionale.

\begin{tabular}{|c|c|c|c|c|c|c|c|c|c|c|c|}
\hline & & & \multicolumn{9}{|c|}{ Regione } \\
\hline & & & $\begin{array}{c}\text { SLO - } \\
\text { Litorale }\end{array}$ & $\begin{array}{c}\text { SLO - } \\
\text { Lubiana }\end{array}$ & $\begin{array}{c}\text { SLO - } \\
\text { Scuole } \\
\text { italiane }\end{array}$ & $\begin{array}{l}\text { Total } \\
\text { e SLO }\end{array}$ & $\begin{array}{l}\text { ITA - } \\
\text { FVG }\end{array}$ & $\begin{array}{c}\text { ITA - } \\
\text { Venezi } \\
\text { a, } \\
\text { Ravenn } \\
\text { a }\end{array}$ & $\begin{array}{c}\text { ITA- } \\
\text { Scuole } \\
\text { slovene }\end{array}$ & $\begin{array}{l}\text { Total } \\
\text { e ITA }\end{array}$ & $\begin{array}{c}\text { TOTAL } \\
\mathrm{E}\end{array}$ \\
\hline \multirow{8}{*}{$\begin{array}{l}\text { Insegnament } \\
\text { o in classi } \\
\text { eterogenee } \\
\text { stimola a } \\
\text { ulteriore } \\
\text { sviluppo } \\
\text { professionale } \\
\text { dell'insegnan } \\
\text { te }\end{array}$} & 1 & $\%$ & 4,0 & 0 & 0 & 1,6 & 3,4 & 5,6 & 2,4 & 3,9 & 2,8 \\
\hline & 2 & $\%$ & 6,0 & 4,3 & 9,7 & 6,3 & 6,9 & 1,9 & 11,9 & 6,5 & 6,4 \\
\hline & 3 & $\%$ & 26,0 & 15,2 & 41,9 & 26,0 & 8,6 & 31,5 & 28,6 & 22,1 & 23,8 \\
\hline & 4 & $\%$ & 30,0 & 32,6 & 22,6 & 29,1 & $\begin{array}{r}36, \\
2\end{array}$ & 38,9 & 33,3 & 36,4 & 33,1 \\
\hline & 5 & $\%$ & 34,0 & 45,7 & 19,4 & 34,6 & $\begin{array}{r}44 \\
8\end{array}$ & 20,4 & 16,7 & 28,6 & 31,3 \\
\hline & Senza risposta & $\%$ & 0 & 2,2 & 6,5 & 2,4 & 0 & 1,9 & 7,1 & 2,6 & 2,5 \\
\hline & TOTALE & $\%$ & 100,0 & 100,0 & 100,0 & $\begin{array}{r}100, \\
0\end{array}$ & $\begin{array}{r}100 \\
, 0\end{array}$ & 100,0 & 100,0 & $\begin{array}{r}100 \\
0\end{array}$ & 100,0 \\
\hline & & $\mathrm{N}$ & 50 & 46 & 31 & 127 & 58 & 54 & 42 & 154 & 281 \\
\hline
\end{tabular}

Tabella 1: Opinioni degli insegnanti sulla relazione tra il lavoro in classi eterogenee e il bisogno di ulteriore sviluppo professionale (\%). 
Dal confronto tra le regioni emerge una stretta relazione tra il lavoro in classi eterogenee e il bisogno di ulteriore aggiornamento e sviluppo professionale tra gli insegnanti della Slovenia centrale e della regione Friuli-Venezia Giulia che si sono dichiarati molto d'accordo $(45,7 \%$ e $44,8 \%$ rispettivamente) e d'accordo $(32,6 \%$ e $36,2 \%$ rispettivamente) con l'affermazione. Una situazione opposta si nota nelle scuole minoritarie sia in Slovenia che in Italia con appena 19,4\% e $16,7 \%$ rispettivamente delle risposte 'completamente d'accordo' e con percentuali molto alte di insegnanti che si sono dichiarati indecisi (41,9\% e $28,6 \%$ rispettivamente). Le scuole minoritarie in entrambe le regioni hanno una lunga tradizione e sono di tipo aperto, accogliendo sia allievi appartenenti alla minoranza che allievi di altre etnie, per cui il lavoro in classi eterogenee può essere considerato una caratteristica inerente di queste scuole da sempre e il bisogno di ulteriore aggiornamento professionale da parte degli insegnanti meno pronunciato rispetto alle regioni con presenza di allievi di diverso background linguistico-culturale più recente.

Lo sviluppo professionale dell'insegnante si riconduce a diverse strutture, tra cui la collaborazione tra le scuole e le università, collaborazione e reti di insegnanti, gruppi di studio e di lavoro e simili che permettono all'insegnante di imparare a gestire il proprio sviluppo professionale e quindi di diventare costruttori del proprio sapere piuttosto che semplici riceventi di informazioni mediate da altri.

La collaborazione tra insegnanti. La collaborazione tra insegnanti è una delle strutture citate nel capoverso precedente che permettono lo sviluppo professionale dell'insegnante. Le risposte degli insegnanti coinvolti nella ricerca mostrano una distribuzione quasi equa tra le risposte 'completamente d'accordo' (26,0\%), 'd'accordo' $(29,2 \%)$ e 'indeciso' $(29,2 \%)$, come si legge nella colonna 'Totale' della tabella 2 .

\begin{tabular}{|c|c|c|c|c|c|c|c|c|c|c|c|}
\hline & & & \multicolumn{9}{|c|}{ Regione } \\
\hline & & & $\begin{array}{l}\text { SLO - } \\
\text { Litoral } \\
\text { e }\end{array}$ & $\begin{array}{c}\text { SLO - } \\
\text { Lubian } \\
\text { a }\end{array}$ & $\begin{array}{l}\text { SLO - } \\
\text { Scuole } \\
\text { italian } \\
\text { e }\end{array}$ & $\begin{array}{l}\text { Total } \\
\text { e SLO }\end{array}$ & $\begin{array}{l}\text { ITA - } \\
\text { FVG }\end{array}$ & $\begin{array}{c}\text { ITA - } \\
\text { Venezia } \\
\text { ' } \\
\text { Ravenn } \\
\text { a }\end{array}$ & $\begin{array}{l}\text { ITA - } \\
\text { Scuole } \\
\text { sloven } \\
\text { e }\end{array}$ & $\begin{array}{l}\text { Total } \\
\text { e ITA }\end{array}$ & $\begin{array}{c}\text { TOTAL } \\
\text { E }\end{array}$ \\
\hline \multirow{6}{*}{$\begin{array}{l}\text { Insegnament } \\
\text { o in classi } \\
\text { eterogenee } \\
\text { promuove la } \\
\text { collaborazion } \\
\text { e tra } \\
\text { insegnanti }\end{array}$} & 1 & $\%$ & 0 & 0 & 3,2 & 8 & 6,9 & 0 & 4,8 & 3,9 & 2,5 \\
\hline & 2 & $\%$ & 10,0 & 2,2 & 16,1 & 8,7 & 12,1 & 14,8 & 2,4 & 10,4 & 9,6 \\
\hline & 3 & $\%$ & 16,0 & 8,7 & 48,4 & 21,3 & 34,5 & 46,3 & 23,8 & 35,7 & 29,2 \\
\hline & 4 & $\%$ & 46,0 & 30,4 & 16,1 & 33,1 & 19,0 & 22,2 & 40,5 & 26,0 & 29,2 \\
\hline & 5 & $\%$ & 26,0 & 56,5 & 12,9 & 33,9 & 25,9 & 11,1 & 21,4 & 19,5 & 26,0 \\
\hline & $\begin{array}{l}\text { Senza } \\
\text { rispost } \\
\text { a }\end{array}$ & $\%$ & 2,0 & 2,2 & 3,2 & 2,4 & 1,7 & 5,6 & 7,1 & 4,5 & 3,6 \\
\hline
\end{tabular}




\begin{tabular}{rrrrrrrrrrr}
\hline TOtALE & $\%$ & 100,0 & 100,0 & 100,0 & 100,0 & 100, & 100,0 & 100,0 & 100,0 & 100,0 \\
& & & & & & 0 & & & & \\
& $\mathrm{~N}$ & 50 & 46 & 31 & 127 & 58 & 54 & 42 & 154 & 281 \\
\hline \hline
\end{tabular}

Tabella 2: Opinioni degli insegnanti sulla relazione tra il lavoro in classi eterogenee e la collaborazione tra insegnanti (\%).

Dal confronto tra le regioni emerge che l'argomento della diversità in classe venga discusso soprattutto nelle scuole slovene, sia in Slovenia che in Italia. L'opinione che il lavoro in classi eterogenee promuova la collaborazione tra insegnanti appare predominante nella regione slovena centrale (86,9\%), nelle scuole slovene sul Litorale sloveno $(72,0 \%)$ e nelle scuole della minoranza slovena in Italia (61,9\%).

Gli insegnanti delle scuole della minoranza italiana in Slovenia, della regione Friuli-Venezia Giulia e nella parte centrale dell'Italia si sono invece dichiarati maggiormente indecisi sulla relazione tra il lavoro con allievi di diverso retroterra linguistico-culturale e la collaborazione tra insegnanti $(48,4 \%, 34,5 \%$ e 46,3\% rispettivamente). In queste regioni si è registrata una percentuale piuttosto accentuata di risposte 'non d'accordo' e 'per niente d'accordo': 19,3\%, 19,0\% e $14,8 \%$ rispettivamente, una percentuale in netto contrasto con $10,0 \%$ di risposte sul Litorale sloveno, 7,2\% nelle scuole slovene in Italia e 2,2\% nella regione centrale della Slovenia. La differenza riscontrata potrebbe essere attribuita a una diversa cultura organizzativa delle scuole e di istituzioni che offrono sostegno professionale agli insegnanti in Italia e Slovenia. II sistema sloveno è organizzato a livello nazionale e promuove la collaborazione tra insegnanti in forme diverse, tra cui gruppi di studio e lavoro, la costruzione di reti di insegnanti, scambi internazionali di insegnanti e allievi per menzionarne solo alcune. D'altra parte, in Italia le opportunità di collaborazione tra insegnanti appaiono più frammentarie e disorganiche, affidate all'iniziativa di varie associazioni, centri ed enti simili. Dato che il contesto educativo è continuamente costretto a rispondere alle esigenze didattiche sempre diverse, sarebbe opportuno dedicarsi alla promozione più ampia possibile della collaborazione tra insegnanti a tutti i livelli: all'interno della scuola, tra le scuole di una regione o un paese, a livello internazionale, perché gli insegnanti avessero l'opportunità di discutere di problemi condivisi, scambiarsi idee, soluzioni a problemi e buone prassi.

Lo sviluppo personale e culturale dell'insegnante. II collegamento tra gli atteggiamenti e convinzioni degli insegnanti sulla diversità razziale, linguistica, culturale ed etnica da una parte e 
dall'altra la loro condotta didattica in termini di comportamenti e decisioni in classe è ormai indiscutibile. Gli insegnanti che hanno (avuto) un limitato contatto con la diversità hanno spesso percezioni distorte e opinioni basate sulla disinformazione filtrata dai media. Tali atteggiamenti e convinzioni distorti interferiscono con l'insegnamento e l'apprendimento invece di facilitarli (Gay 144). Secondo Spiteri (12) qualsiasi contatto con la diversità è un'opportunità per la mediazione e lo sviluppo della sensibilità interculturale, a patto che, osserva Gay (144) non venga ignorato o respinto come non importante. Consideriamo pertanto significativamente positivo il giudizio degli insegnanti coinvolti nella ricerca sulla relazione tra il lavoro in classi eterogenee e il loro sviluppo personale e culturale. I risultati nella tabella 3 rivelano che quasi la metà di insegnanti interpellati $(42,7 \%)$ si è dichiarata molto d'accordo sulla relazione tra l'insegnamento in classi eterogenee e lo sviluppo personale e culturale dell'insegnante, un terzo $(32,0 \%)$ hanno risposto di essere d'accordo con l'affermazione e solo un quinto $(19,2 \%)$ degli insegnanti appare indeciso.

\begin{tabular}{|c|c|c|c|c|c|c|c|c|c|c|c|}
\hline & & & \multicolumn{9}{|c|}{ Regione } \\
\hline & & & $\begin{array}{l}\text { SLO - } \\
\text { Litoral } \\
\text { e }\end{array}$ & $\begin{array}{c}\text { SLO - } \\
\text { Lubian } \\
\text { a }\end{array}$ & $\begin{array}{c}\text { SLO - } \\
\text { Scuole } \\
\text { italian } \\
\mathrm{e}\end{array}$ & $\begin{array}{l}\text { Total } \\
\text { e SLO }\end{array}$ & $\begin{array}{l}\text { ITA - } \\
\text { FVG }\end{array}$ & $\begin{array}{c}\text { ITA - } \\
\text { Venezia } \\
\text { ' } \\
\text { Ravenn } \\
\text { a }\end{array}$ & $\begin{array}{c}\text { ITA- } \\
\text { Scuole } \\
\text { sloven } \\
\text { e }\end{array}$ & $\begin{array}{l}\text { Total } \\
\text { e ITA }\end{array}$ & $\begin{array}{c}\text { TOTAL } \\
\mathrm{E}\end{array}$ \\
\hline \multirow{8}{*}{$\begin{array}{l}\text { Insegnamento } \\
\text { in classi } \\
\text { eterogenee } \\
\text { promuove lo } \\
\text { sviluppo } \\
\text { personale e } \\
\text { culturale } \\
\text { dell'insegnant } \\
\text { e }\end{array}$} & 1 & $\%$ & 2,0 & 0 & 0 & 8 & 0 & 1,9 & 2,4 & 1,3 & 1,1 \\
\hline & 2 & $\%$ & 2,0 & 4,3 & 3,2 & 3,1 & 1,7 & 3,7 & 4,8 & 3,2 & 3,2 \\
\hline & 3 & $\%$ & 14,0 & 6,5 & 35,5 & 16,5 & 20,7 & 18,5 & 26,2 & 21,4 & 19,2 \\
\hline & 4 & $\%$ & 46,0 & 30,4 & 25,8 & 35,4 & 24,1 & 35,2 & 28,6 & 29,2 & 32,0 \\
\hline & 5 & $\%$ & 36,0 & 56,5 & 32,3 & 42,5 & 53,4 & 38,9 & 33,3 & 42,9 & 42,7 \\
\hline & $\begin{array}{l}\text { Senza } \\
\text { rispost } \\
\text { a }\end{array}$ & $\%$ & 0 & 2,2 & 3,2 & 1,6 & 0 & 1,9 & 4,8 & 1,9 & 1,8 \\
\hline & $\begin{array}{l}\text { TOTAL } \\
\text { E }\end{array}$ & $\%$ & 100,0 & 100,0 & 100,0 & 100,0 & $\begin{array}{r}100 \\
0\end{array}$ & 100,0 & 100,0 & 100,0 & 100,0 \\
\hline & & $\mathrm{N}$ & 50 & 46 & 31 & 127 & 58 & 54 & 42 & 154 & 281 \\
\hline
\end{tabular}

Tabella 3: Opinioni degli insegnanti sulla relazione tra il lavoro in classi eterogenee e lo sviluppo personale e culturale dell'insegnante (\%).

I risultati del confronto tra le regioni sono simili all'affermazione sulla relazione tra il lavoro in classi eterogenee e il bisogno di ulteriore aggiornamento e sviluppo professionale (Tabella 1). Dalla tabella 3 emerge che anche la relazione tra il lavoro con allievi di diversa provenienza e lo sviluppo personale e culturale dell'insegnante viene considerata come molto stretta tra gli insegnanti 
della Slovenia centrale con $56,5 \%$ di risposte 'completamente d'accordo' e 30,4\% di risposte 'd'accordo', e insegnanti della regione Friuli-Venezia Giulia che si sono dichiarati molto d'accordo $(53,4 \%)$ e d'accordo $(24,1 \%)$ con l'affermazione. Il giudizio meno favorevole è stato di nuovo riscontrato tra gli insegnanti delle scuole minoritarie da entrambe le parti del confine.

Segue I'analisi dei risultati relativi alla didattica in classi eterogenee.

Insegnamento in classi eterogenee porta a rallentare lo svolgimento delle attività didattiche. I risultati nella tabella 4 indicano che un terzo degli insegnanti interpellati $(33,4 \%)$ si è dichiarato indeciso sul fatto che in classi eterogenee le attività didattiche vengano svolte più lentamente, mentre sono quasi a pari merito le risposte degli insegnanti che si sono dichiarati d'accordo $(19,9 \%)$ e non d'accordo $(20,6 \%)$.

\begin{tabular}{|c|c|c|c|c|c|c|c|c|c|c|c|}
\hline & & & \multicolumn{9}{|c|}{ Regione } \\
\hline & & & $\begin{array}{c}\text { SLO - } \\
\text { Litoral } \\
\text { e }\end{array}$ & $\begin{array}{l}\text { SLO - } \\
\text { Lubian } \\
\quad \text { a }\end{array}$ & $\begin{array}{c}\text { SLO - } \\
\text { Scuole } \\
\text { italian } \\
\text { e }\end{array}$ & $\begin{array}{l}\text { Total } \\
\text { e SLO }\end{array}$ & $\begin{array}{l}\text { ITA - } \\
\text { FVG }\end{array}$ & $\begin{array}{c}\text { ITA - } \\
\text { Venezia } \\
\text { ' } \\
\text { Ravenn } \\
\text { a }\end{array}$ & $\begin{array}{l}\text { ITA- } \\
\text { Scuole } \\
\text { sloven } \\
\text { e }\end{array}$ & $\begin{array}{l}\text { Total } \\
\text { e ITA }\end{array}$ & $\begin{array}{c}\text { TOTAL } \\
\text { E }\end{array}$ \\
\hline \multirow{8}{*}{$\begin{array}{l}\text { Insegnament } \\
\text { o in classi } \\
\text { eterogenee } \\
\text { porta a } \\
\text { rallentare lo } \\
\text { svolgimento } \\
\text { delle attività } \\
\text { scolastiche }\end{array}$} & 1 & $\%$ & 8,0 & 8,7 & 12,9 & 9,4 & 15,5 & 24,1 & 4,8 & 15,6 & 12,8 \\
\hline & 2 & $\%$ & 22,0 & 37,0 & 6,5 & 23,6 & 19,0 & 18,5 & 16,7 & 18,2 & 20,6 \\
\hline & 3 & $\%$ & 32,0 & 30,4 & 45,2 & 34,6 & 36,2 & 31,5 & 31,0 & 33,1 & 33,8 \\
\hline & 4 & $\%$ & 26,0 & 15,2 & 32,3 & 23,6 & 15,5 & 14,8 & 21,4 & 16,9 & 19,9 \\
\hline & 5 & $\%$ & 12,0 & 6,5 & 0 & 7,1 & 10,3 & 9,3 & 16,7 & 11,7 & 9,6 \\
\hline & $\begin{array}{l}\text { Senza } \\
\text { rispost } \\
\text { a }\end{array}$ & $\%$ & 0 & 2,2 & 3,2 & 1,6 & 3,4 & 1,9 & 9,5 & 4,5 & 3,2 \\
\hline & TOTALE & $\%$ & 100,0 & 100,0 & 100,0 & 100,0 & $\begin{array}{r}100, \\
0\end{array}$ & 100,0 & 100,0 & 100,0 & 100,0 \\
\hline & & $\mathrm{N}$ & 50 & 46 & 31 & 127 & 58 & 54 & 42 & 154 & 281 \\
\hline
\end{tabular}

Tabella 4: Opinioni degli insegnanti sulla relazione tra il lavoro in classi eterogenee e la dinamica dell'attività didattica (\%).

II confronto tra le regioni divide il campione in tre gruppi: (1) gli insegnanti delle scuole minoritarie, (2) gli insegnanti delle scuole maggioritarie sulle aree linguisticamente e culturalmente miste, e (3) gli insegnanti delle scuole nell'area centrale dello stato. I primi osservano uno stretto collegamento tra il lavoro in classi eterogenee e il rallentamento della dinamica nello svolgimento delle attività didattiche. Le loro risposte sono superiori alla media nazionale ${ }^{9}$ con

${ }^{9} 30,7 \%$ in Slovenia e $28,6 \%$ in Italia. 
$32,3 \%$ di risposte nelle scuole italiane in Slovenia e con $38,1 \%$ di risposte nelle scuole slovene in Italia. ${ }^{10}$ È diametralmente contrario il giudizio nelle zone centrali della Slovenia e dell'Italia in cui si sono registrate le più alte risposte negative all'affermazione: $45,7 \%$ in Slovenia e $42,6 \%$ in Italia. ${ }^{11}$ Tra gli insegnanti delle scuole maggioritarie nelle aree mistilingui le risposte sono prevalentemente neutre.

La presenza di allievi di diverso background linguisticoculturale esige indubbiamente una velocità di esecuzione adeguata ai bisogni linguistici di tutti gli allievi, compresi quelli cui la prima lingua non è la lingua dell'istruzione. L'insegnante deve concedere loro più tempo per processare a livello cognitivo e linguistico l'input ricevuto e quindi per rispondere adeguatamente. Gli allievi con diverso background linguistico-culturale possono beneficiare di ripetizioni e riformulazioni dell'input, il che richiede tempo aggiuntivo (Heineke, McTighe 212). Partendo dal presupposto che lo svolgimento delle attività didattiche sia necessariamente più lento in classi eterogenee, risulta interessante la discrepanza tra le risposte riferite dagli insegnanti interpellati. La varianza potrebbe essere attribuita al numero di allievi nella classe, alla percentuale di allievi con diverso background linguistico-culturale, al grado di diversità della loro provenienza. In classi numerose con alta presenza di allievi con diverso background linguistico-culturale che provengono da una sostanziale varietà di contesti, soprattutto se si tratta di contesti geograficamente e/o culturalmente lontani dalla cultura ospite, il bisogno di adattare la didattica, compreso il rallentamento dello svolgimento delle attività didattiche, sarà maggiore rispetto ad altre realtà educative.

II lavoro in classi eterogenee esige dall'insegnante un carico di lavoro maggiore. Quasi tre quarti degli insegnanti coinvolti nella ricerca $(73,7 \%)$ concorda con l'affermazione. II confronto tra le regioni mostra che il carico del lavoro aggiuntivo è stato osservato più dagli insegnanti in Slovenia $(82,7 \%)$ che gli insegnanti in Italia $(66,2 \%)$. II giudizio meno favorevole all'affermazione è stato di nuovo espresso dagli insegnanti delle scuole minoritarie in entrambi i paesi (Tabella 5).

\footnotetext{
${ }^{10}$ Tutte le percentuali indicate sono la somma delle percentuali di risposte 'assolutamente d'accordo' e 'd'accordo'.

${ }^{11}$ Le percentuali indicate sono la somma delle percentuali di risposte 'per niente d'accordo' e 'non d'accordo'.
} 


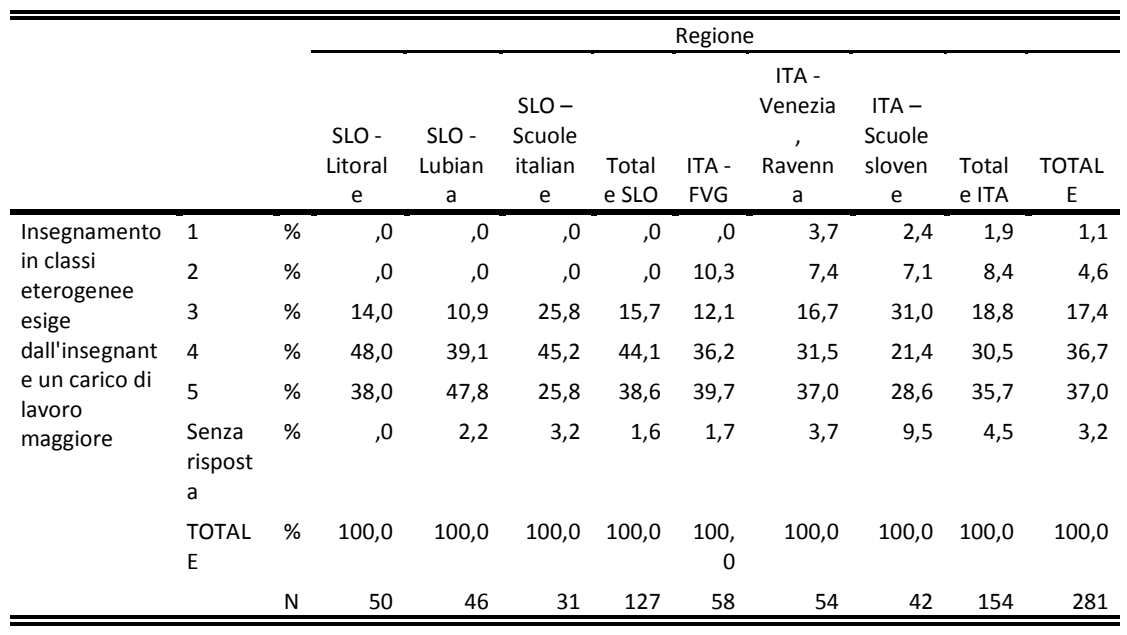

Tabella 5: Opinioni degli insegnanti sulla relazione tra il lavoro in classi eterogenee e carico di lavoro aggiuntivo (\%).

L'eterogeneità linguistico-culturale della classe richiede senza dubbio un carico di lavoro maggiore, sia nella fase della progettazione, che durante le lezioni e nella fase della valutazione. II lavoro aggiuntivo consiste nella costruzione di materiale aggiuntivo, adattamento dell'input e della valutazione. II problema sembra più pronunciato in classi numerose, con allievi più grandi e nelle materie umanistiche e l'arte (Atkins, Carter, Nichol 3).

L'insegnamento in classi eterogenee è condizionato dai problemi di comunicazione tra gli allievi. Dalle risposte degli insegnanti interpellati emerge che gli eventuali problemi di comunicazione tra allievi parlanti lingue diverse non hanno particolare impatto negativo sullo svolgimento delle attività didattiche. Nella tabella 6 si legge che quasi la metà degli insegnanti si è dichiarata di non essere d'accordo con l'affermazione $(42,7 \%)$, che un terzo è apparso indeciso $(32,7 \%)$ e solo un quinto degli insegnanti afferma che $\mathrm{i}$ problemi di comunicazione tra allievi interferisce con il processo didattico in classe. Il giudizio meno favorevole si è riscontrato nella regione della Slovenia centrale con solo $10,8 \%$ di insegnanti che conferma la relazione tra $\mathrm{i}$ problemi di comunicazione tra gli allievi e i problemi nello svolgimento delle attività didattiche, mentre il giudizio più favorevole è stato espresso dagli insegnanti delle scuole italiane in Slovenia con $38,8 \%$ di risposte affermative. 


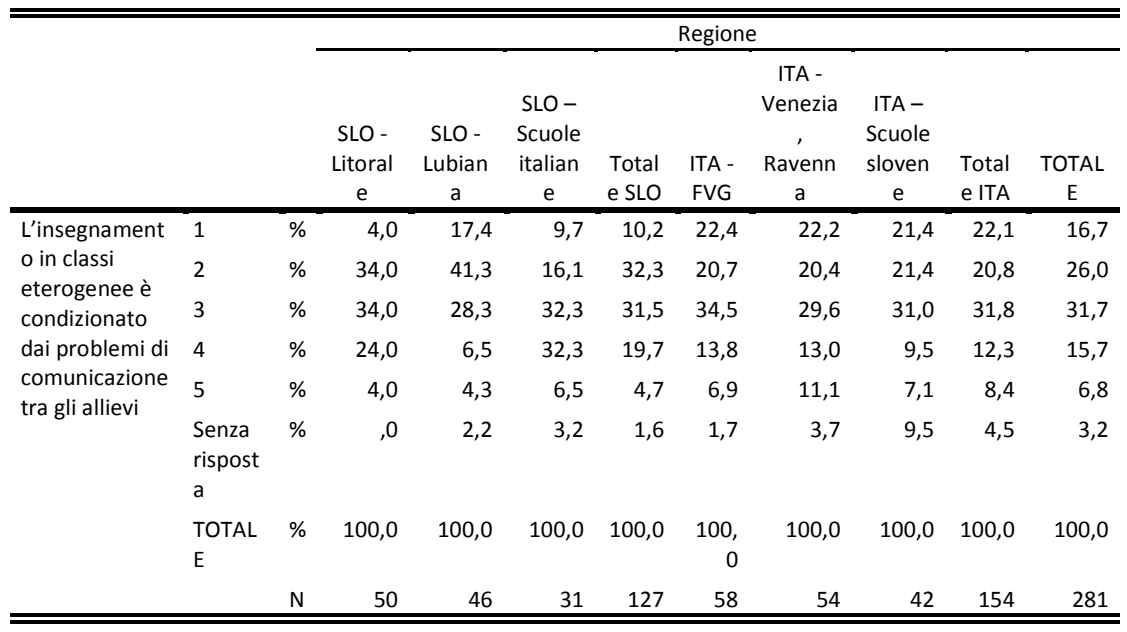

Tabella 6: Opinioni degli insegnanti sulla relazione tra il lavoro in classi eterogenee e i problemi di comunicazione tra gli allievi (\%).

L'insegnamento in classi eterogenee porta ad abbassare il livello di competenza della lingua d'insegnamento di tutti gli allievi. La distribuzione delle risposte (Tabella 7) rispecchia quella alla domanda precedente (Tabella 6). Gli insegnanti delle scuole minoritarie sul Litorale sloveno si sono dichiarati maggiormente favorevoli alle affermazioni che l'insegnamento in classi eterogenee è condizionato dai problemi di comunicazione tra gli allievi e che porta ad abbassare il livello di competenza nella lingua d'insegnamento. Del tutto opposte si sono rivelate le risposte degli insegnanti dell'area centrale della Slovenia che si sono dichiarati come meno favorevoli alle due affermazioni. Con l'affermazione che l'insegnamento in classi eterogenee porti ad abbassare il livello della competenza nella lingua d'insegnamento non sono stati d'accordo inoltre gli insegnanti dell'area centrale dell'Italia (Venezia e Ravenna). Risposte leggermente più favorevoli sono state riferite dagli insegnanti delle scuole maggioritarie da entrambe le parti del confine, mentre le più favorevoli sono state registrate nelle scuole minoritarie in entrambi i paesi, con le scuole italiane sul Litorale sloveno significativamente più favorevole rispetto a tutte le scuole coinvolte nella ricerca. 


\begin{tabular}{|c|c|c|c|c|c|c|c|c|c|c|c|}
\hline & & & \multicolumn{9}{|c|}{ Regione } \\
\hline & & & $\begin{array}{l}\text { SLO - } \\
\text { Litoral } \\
\text { e }\end{array}$ & $\begin{array}{l}\text { SLO - } \\
\text { Lubian } \\
\text { a }\end{array}$ & $\begin{array}{c}\text { SLO - } \\
\text { Scuole } \\
\text { italian } \\
\text { e }\end{array}$ & $\begin{array}{l}\text { Total } \\
\text { e SLO }\end{array}$ & $\begin{array}{l}\text { ITA - } \\
\text { FVG }\end{array}$ & $\begin{array}{c}\text { ITA - } \\
\text { Venezia } \\
\quad, \\
\text { Ravenn } \\
\text { a }\end{array}$ & $\begin{array}{l}\text { ITA- } \\
\text { Scuole } \\
\text { sloven } \\
\text { e }\end{array}$ & $\begin{array}{l}\text { Total } \\
\text { e ITA }\end{array}$ & $\begin{array}{c}\text { TOTAL } \\
\text { E }\end{array}$ \\
\hline \multirow{8}{*}{$\begin{array}{l}\text { L'insegnament } \\
\text { o in classi } \\
\text { eterogenee } \\
\text { porta ad } \\
\text { abbassare il } \\
\text { livello di } \\
\text { competenza } \\
\text { della lingua } \\
\text { d'insegnament } \\
\text { o di tutti gli } \\
\text { allievi }\end{array}$} & 1 & $\%$ & 26,0 & 32,6 & 6,5 & 23,6 & 39,7 & 51,9 & 16,7 & 37,7 & 31,3 \\
\hline & 2 & $\%$ & 38,0 & 39,1 & 12,9 & 32,3 & 24,1 & 16,7 & 21,4 & 20,8 & 26,0 \\
\hline & 3 & $\%$ & 14,0 & 13,0 & 32,3 & 18,1 & 17,2 & 14,8 & 21,4 & 17,5 & 17,8 \\
\hline & 4 & $\%$ & 18,0 & 10,9 & 22,6 & 16,5 & 13,8 & 5,6 & 21,4 & 13,0 & 14,6 \\
\hline & 5 & $\%$ & 4,0 & 2,2 & 22,6 & 7,9 & 3,4 & 7,4 & 9,5 & 6,5 & 7,1 \\
\hline & $\begin{array}{l}\text { Senza } \\
\text { rispost } \\
\text { a }\end{array}$ & $\%$ & 0 & 2,2 & 3,2 & 1,6 & 1,7 & 3,7 & 9,5 & 4,5 & 3,2 \\
\hline & $\begin{array}{l}\text { TOTAL } \\
\text { E }\end{array}$ & $\%$ & 100,0 & 100,0 & 100,0 & 100,0 & $\begin{array}{r}100 \\
0\end{array}$ & 100,0 & 100,0 & 100,0 & 100,0 \\
\hline & & $\mathrm{N}$ & 50 & 46 & 31 & 127 & 58 & 54 & 42 & 154 & 281 \\
\hline
\end{tabular}

Tabella 7: Opinioni degli insegnanti sulla relazione il lavoro in classi eterogenee e il livello di competenza comunicativa nella lingua d'insegnamento di tutti gli allievi (\%).

Rhodes, Ochoa e Ortiz (179-184) fanno notare le difficoltà nella valutazione della competenza in lingua della scuola degli allievi di diverso background linguistico-culturale, dovute principalmente agli strumenti a disposizione. Mentre gli insegnanti dispongono di numerosi strumenti per la valutazione di abilità cognitive e intellettive, nessuno degli strumenti per la valutazione della competenza sia della lingua d'origine sia della lingua d'insegnamento si basa su norme che siano del tutto appropriate per gli allievi di cui la lingua d'insegnamento è la seconda lingua, nemmeno nel caso che abbiano raggiunto un'ottima padronanza in questa lingua. Di conseguenza, $i$ risultati che emergono dalla valutazione della competenza comunicativa nella lingua d'insegnamento tendono a rappresentare le abilità degli allievi di diversa origine linguistico-culturale in misura insufficiente e decisamente minore rispetto alle loro effettive abilità. Fanno eccezione, secondo gli autori, gli allievi che hanno ricevuto insegnamento sistematico nella loro lingua madre.

In passato i paesi europei si sono dedicati alla cura della lingua d'origine degli allievi di diverso background, ma gradualmente questa attenzione andava diminuendosi e in alcuni paesi d'Europa oggi prevale una visione integralista (Pulinx, Van Avermaet 10). La precedenza per la lingua d'insegnamento ha origine nelle ricerche comparative internazionali, per esempio PISA, che hanno dimostrato risultati significativamente minori tra gli allievi parlanti una lingua diversa da quella d'insegnamento rispetto agli allievi parlanti nativi di 
quella lingua (Pulinx, Van Avermaet 16-17). Dalle ricerche di Rhodes, Ochoa e Ortiz emerge che la valutazione della competenza nella lingua d'insegnamento è molto più complessa di una semplice verifica nelle abilità della comprensione letta. Gli studiosi suggeriscono che dovrebbe coinvolgere una batteria di test, compresi test di abilità cognitive e intellettive (179).

Le percezioni che gli insegnanti hanno sullo sviluppo della competenza comunicativa nella lingua d'insegnamento di tutti gli allievi, nonché le loro preoccupazioni per il profitto scolastico sia di allievi parlanti una lingua diversa dalla lingua d'insegnamento sia gli allievi parlanti la lingua della scuola sono importanti perché incidono sulle loro scelte e azioni didattiche, sulla loro comunicazione in classe e con i genitori. Si dovrebbe pertanto dedicare maggiore attenzione a rendere consapevoli gli insegnanti della complessità del processo non solo di apprendimento ma anche di valutazione della competenza comunicativa e proporre loro soluzioni che vadano oltre la verifica delle abilità linguistico-comunicative. Le scuole che ne avrebbero maggiore bisogno, secondo i risultati emersi dalla nostra ricerca, sono le scuole minoritarie. Frequentate da allievi di varia origine linguistica, con potenzialmente classi in cui i parlanti nativi sono in minoranza, le scuole minoritarie devono spesso seguire itinerari didattici talmente diversificati che creano negli insegnanti la frustrazione di non poter seguire i singoli allievi nella misura in cui vorrebbero, il che può condurre alla sensazione che l'attenzione dedicata alla differenziazione sia a detrimento di un adeguato sviluppo della competenza nella lingua d'insegnamento.

L'impatto della presenza di allievi di lingue e culture diverse sullo sviluppo di buone capacità relazionali tra gli allievi. La maggioranza degli insegnanti ha dichiarato che l'eterogeneità linguistica e culturale promuove lo sviluppo di buone capacità relazionali tra gli allievi: 54,1\% degli insegnanti ha dichiarato che l'impatto della presenza di allievi di diverse lingue culture in classe è positiva e per $20,3 \%$ degli insegnanti è addirittura molto positiva.

II giudizio più favorevole è emerso dalle risposte degli insegnanti dell'area centrale della Slovenia con solo $8,7 \%$ di insegnanti indecisi e nessuno sfavorevole. Seguono gli insegnanti della regione Friuli-Venezia Giulia con 15,5\% di insegnanti indecisi e 5,2\% sfavorevoli, gli insegnanti delle scuole italiane sul Litorale sloveno con $16,1 \%$ di indecisi e 3,2\% di sfavorevoli, gli insegnanti delle scuole a Venezia e a Ravenna con $29,6 \%$ di insegnanti indecisi e $1,9 \%$ sfavorevoli e infine gli insegnanti delle scuole slovene sul Litorale sloveno con $32,0 \%$ di indecisi e 4,0\% di sfavorevoli. 


\begin{tabular}{|c|c|c|c|c|c|c|c|c|c|c|c|}
\hline & & & \multicolumn{9}{|c|}{ Regione } \\
\hline & & & $\begin{array}{l}\text { SLO - } \\
\text { Litorale }\end{array}$ & $\begin{array}{c}\text { SLO - } \\
\text { Lubiana }\end{array}$ & $\begin{array}{l}\text { SLO - } \\
\text { Scuole } \\
\text { italiane }\end{array}$ & $\begin{array}{c}\text { Totale } \\
\text { SLO }\end{array}$ & $\begin{array}{l}\text { ITA - } \\
\text { FVG }\end{array}$ & $\begin{array}{c}\text { ITA - } \\
\text { Venezia, } \\
\text { Ravenna }\end{array}$ & $\begin{array}{c}\text { ITA- } \\
\text { Scuole } \\
\text { slovene }\end{array}$ & $\begin{array}{l}\text { Totale } \\
\text { ITA }\end{array}$ & TOTALE \\
\hline \multirow{7}{*}{$\begin{array}{l}\text { L'impatto } \\
\text { della } \\
\text { presenza } \\
\text { di allievi } \\
\text { di lingue e } \\
\text { culture } \\
\text { diverse } \\
\text { sullo } \\
\text { sviluppo } \\
\text { di buone } \\
\text { capacità } \\
\text { relazionali } \\
\text { è }\end{array}$} & Negativo & $\%$ & 4,0 & 0 & 3,2 & 2,4 & 5,2 & 1,9 & 2,4 & 3,2 & 2,8 \\
\hline & $\begin{array}{l}\text { Né } \\
\text { negativo, } \\
\text { né } \\
\text { positivo }\end{array}$ & $\%$ & 32,0 & 8,7 & 16,1 & 19,7 & 15,5 & 29,6 & 26,2 & 23,4 & 21,7 \\
\hline & Positivo & $\%$ & 50,0 & 54,3 & 61,3 & 54,3 & 53,4 & 51,9 & 57,1 & 53,9 & 54,1 \\
\hline & $\begin{array}{l}\text { Molto } \\
\text { positivo }\end{array}$ & $\%$ & 12,0 & 37,0 & 16,1 & 22,0 & 25,9 & 16,7 & 11,9 & 18,8 & 20,3 \\
\hline & $\begin{array}{l}\text { Senza } \\
\text { risposta }\end{array}$ & $\%$ & 2,0 & 0 & 3,2 & 1,6 & 0 & 0 & 2,4 & 6 & 1,1 \\
\hline & TOTALE & $\%$ & 100,0 & 100,0 & 100,0 & 100,0 & 100,0 & 100,0 & 100,0 & 100,0 & 100,0 \\
\hline & & $\mathrm{N}$ & 50 & 46 & 31 & 127 & 58 & 54 & 42 & 154 & 281 \\
\hline
\end{tabular}

Tabella 9: Opinioni degli insegnanti sulla relazione tra la presenza di allievi di lingue e culture diverse e lo sviluppo di buone capacità relazionali tra gli allievi (\%).

\section{Conclusioni}

Le percezioni che gli insegnanti hanno del proprio lavoro hanno origine nella loro preparazione didattica per il lavoro in classe, in una naturale disposizione personale per la professione, e nel senso di realizzazione professionale e personale. La conoscenza delle percezioni degli insegnanti permette di programmare interventi di vario genere: dalla formazione dei futuri insegnanti, degli insegnanti in servizio, alla preparazione di linee guida per gli insegnanti e di materiale didattico in modo da soddisfare i bisogni educativi in continua trasformazione.

Gli insegnanti con una percezione positiva del proprio lavoro sono maggiormente motivati per il lavoro in classe, per l'adattamento della loro didattica alle diverse realtà cui fanno fronte, per l'aggiornamento continuo in vari aspetti legati alla professione dell'insegnante, per lo scambio di esperienze con altri insegnanti, e per la partecipazione ai progetti di ricerca.

Gli insegnanti coinvolti nella nostra ricerca insegnano in classi linguisticamente e culturalmente eterogenee e dichiarano che questa esperienza professionale rappresenti per loro un'occasione e una motivazione a continuare a crescere professionalmente. Tra le varie strutture, sottostanti lo sviluppo professionale degli insegnanti, sembra che la cooperazione e lo scambio di esperienze con altri insegnanti non sia privilegiato. In qualità di formatori di attuali e futuri insegnanti dovremmo quindi dedicarci alla promozione attiva della collaborazione tra insegnanti perché questi continuino ad essere in grado di rispondere alle esigenze didattiche sempre diverse, dovute ai continui 
cambiamenti nel contesto sociale e educativo. L'opportunità di discutere problemi condivisi, scambiarsi idee, soluzioni ai problemi e buone prassi permette agli insegnanti di discutere su esperienze e su problemi in comune con insegnanti in simili contesti didattici e soprattutto di riuscire a adeguarsi alle nuove esperienze e problemi, il che si trova alla base dello stesso sviluppo professionale dell'insegnante.

Un insegnante bene formato e informato comprende che la presenza di allievi di diverse provenienze linguistico-culturali (1) porta a rallentare lo svolgimento delle attività didattiche, soprattutto in classi numerose con alta presenza di allievi con diverso background linguistico-culturale che provengono da una sostanziale varietà di contesti, in particolare se si tratta di contesti geograficamente e/o culturalmente lontani dalla cultura ospite; (2) richiede un carico di lavoro maggiore in tutte le fasi del processo didattico e che il problema sembri più rilevante in classi numerose, con allievi più grandi e nelle materie umanistiche e l'arte (Atkins, Carter,Nichol 3); (3) può condizionare il lavoro in classe per via di problemi di comunicazione tra allievi; e che (4) può contribuire alla percezione che porti ad abbassare il livello di competenza nella lingua della scuola di tutti gli allievi.

La partecipazione degli insegnanti alle varie strutture che sostengono lo sviluppo professionale dell'insegnante permette loro di riflettere su diverse problematiche legate al lavoro in classi eterogenee e soprattutto di comprendere la loro complessità. La percezione dello sviluppo della competenza nella lingua della scuola, per esempio, è legata quasi esclusivamente ai risultati della verifica e valutazione linguistica, che tuttavia in determinati casi non riesce a rappresentare adeguatamente le competenze degli allievi, in particolare degli allievi parlanti una lingua diversa dalla lingua della scuola, i cui risultati spesso risultano decisamente minori rispetto alle loro effettive abilità. La consapevolezza della complessità del problema della valutazione linguistica e la conoscenza delle potenzialità della sua risoluzione conducono indubbiamente a una percezione diversa del profitto scolastico degli allievi e di conseguenza a una minore frustrazione professionale negli insegnanti.

Sebbene dall'inchiesta risulti che gli insegnanti interpellati abbiano una percezione positiva del proprio sviluppo professionale e del lavoro in classi eterogenee riteniamo necessario ribadire l'importanza della loro motivazione a continuare a crescere professionalmente che è in parte legata anche al loro ambiente lavorativo. Se in sintonia con la filosofia didattica degli insegnanti e la loro personalità che si manifesta soprattutto nel rispetto e nell'empatia 
verso tutti gli allievi, gli insegnanti continueranno a essere efficaci e motivati allo sviluppo professionale.

\section{Bibliografia:}

Atkins, John, David Carter, e Mike Nichol. Impact of Class Size on Teacher Workload. Report December 2002. Dublin: National Union of Teachers, 2002. 10 agosto 2019.

Bennett, Janet M., e Milton J. Bennett. "Developing Intercultural Sensitivity: An Integrative Approach to Global and Domestic Diversity." The Diversity Symposium. Portland, Oregon: The Intercultural Communication Institute, 2001. 4 giugno 2003.

Dong, Ren Yu. "Preparing Secondary Subject Area: Teachers to Teach Linguistically and Culturally Diverse Students." The Clearing House: A Journal of Educational Strategies, Issues and Ideas 7 agosto 2010: (77)5, 202-208. Taylor and Francis Online. 7 marzo 2019.

Farber, Barry A. "Understanding and treating burnout in a changing culture." Journal of Clinical Psychology aprile 2000: 56, 589594.

Galbraith, Paul, e Kris Anstrom. "Peer Coaching: An Effective Staff Development Model for Educators of Linguistically and Culturally Diverse Students." Directions in Language and Education primavera 1995: (1) 3, 3-10. ERIC. 10 agosto 2019.

Garmon, Arthur M. "Changing Preservice Teachers' Attitudes/Beliefs About Diversity: What are the Critical Factors?" Journal of Teacher Education $1^{\circ}$ maggio 2004: (55) 3, 201-213. SAGE. 2 aprile 2018.

Gay, Geneva. "Acting on Beliefs in Teacher Education for Cultural Diversity." Journal for Teacher Education gennaio/febbraio 2010: (1-2) 61, 143-152. ERIC. 10 agosto 2019.

Heineke, Amy J., e Jay McTighe. Using Understanding by Design in the Culturally and Linguistically Diverse Classroom. Alexandria, VA: ASCD, 2018.

Knight, Stephanie L., e Donna L. Wiseman. "Professional Development for Teachers of Diverse Students: A Summary of the Research." Journal of Education for Students Placed at Risk (JESPAR) 16 novembre 2009: (10) 4, 387-405. Taylor and Francis Online. 7 marzo 2019.

"Lessons Learned from a Research Synthesis on the Effects of Teachers' Professional Development on Culturally Diverse 
Students." Preparing Quality Educators for English Language Learners: Research, Policy and Practice. Ed. Téllez, Kip e Hersh C. Waxman. New York: Routhledge, 2006. Ladson-Billings, Gloria J. Preparing Teachers for Diverse Student Populations: "A Critical Race Theory Perspective." Review of Research in Education $1^{\circ}$ gennaio 1999: 24, 211-247. JSTOR. 7 marzo 2019.

Lehman, Cheryl L. Multicultural competence: “A Literature Review Supporting Focused Training for Preservice Teachers Teaching Diverse Students." Journal of Education and Practice 2017: (8) 10, 109-116. ERIC. 7 febbraio 2019.

McAllister, Gretchen F., e Jacqueline Jordan Irvine. "Cross Cultural Competency and Multicultural Teacher Education." Review of Educational Research $1^{\circ}$ marzo 2000: (70) 1, 3-24. Elsevier. 16 febbraio 2004.

Moon, Tonya R., Carolyn M. Callahan, e Carol A. Tomlinson. "The Effects of Mentoring Relationships on Preservice Teachers' Attitudes Toward Academically Diverse Students." Gifted Child Quarterly $1^{\circ}$ aprile 1999: (43) 2, 56-62. SAGE. 2 aprile 2018.

Pederson, Patricia V. "Intercultural Sensitivity and the Early Adolescent." Annual Conference of the National Council for the Social Studies. Cincinnati, Ohio: National Council for the Social Studies, 1997. 15 settembre 2015.

Pohan, Cathy A. "Preservice Teachers' Beliefs About Diversity: Uncovering Factors Leading to Multicultural Responsiveness." Equity and Excellence in Education 9 luglio 2006: (29) 3, 62-69. Taylor and Francis Online. 7 marzo 2019.

Pulinx, Reinhilde, e Piet Van Avermaet. "Linguistic diversity and education. Dynamic interaction between language education policies and teachers' beliefs. A qualitative study in secondary schools in Flanders (Belgium)." Revue française de linguistique appliquée dicembre 2014: (2) 14, 9-27. CAIRN. 6 luglio 2019.

Rhodes, Robert L., Salvador Hector Ochoa, e Samuel O. Ortiz. Assessing Culturally and Linguistically Diverse Students: A Practical Guide. New York: Guilford, 2005.

Sleeter, Christine. "Preparing White Teachers for Diverse Students." Handbook on Research on Teacher Education: Enduring Questions in Changing Contexts. $3^{\mathrm{a}}$ ed. Ed. Cochran-Smith, Marilyn, Sharon Feiman-Nemser, D. John Mclntyre, Kelly E. Demers. New York: Routhledge, 2008. Sleeter, Christine. "Preparing Teachers for Culturally Diverse Schools: Research and the Overwhelming Presence of Whiteness. " Journal of 
Teacher Education $1^{\circ}$ marzo 2001: (2) 52, 94-106. SAGE.. 7 marzo 2019.

Spiteri, Damian. Multiculturalism, Higher Education and Intercultural Communication. Developing Strengths-Based Narrarives for Teaching and Learning. London: Macmillan, 2017.

Tatar, Moshe, e Gabriel Horenczyk. "Diversity-related burnout among teachers." Teaching and teaching education maggio 2003: (4) 19, 397-408. ERIC. 7 febbraio 2019.

Tatto, Maria Teresa. "Examining Values and Beliefs About Teaching Diverse Students: Understanding the Challenges for Teacher Education." Educational Evaluation and Policy Analysis $1^{\circ}$ giugno 2018: (18) 2, 155-180. SAGE. 2 aprile 2018.

Tucker, Carolyn M., Terrence Porter, Wendy M. Reinke, Keith C. Herman, Phyllis D. Ivery, Christopher E. Mack, e Erin S. Jackson. "Promoting Teacher Efficacy for Working with Culturally Diverse Students." Preventing School Failure: Alternative Education for Children and Youth agosto 2010: (1) 50, 29-34. Science and Education. 18 settembre 2015.

Walker, Robert J. "Twelve Characteristics of an Effective Teacher: A Longitudinal, Qualitative, Quasi-Research Study of In-service and Pre-service Teachers' Opinions." Educational Horizons autunno 2008: (87) 1, 61-68. ERIC. 7 febbraio 2019.

Zorman, Anja, e Nives Zudič Antonič. "Intercultural sensitivity of teachers." Annales, Series Historia et Sociologia 2019: (2) 29, 247-258. DOI 10.19233/ASHS.2019.17.

Zudič Antonič, Nives. "L'educazione letteraria per lo sviluppo della consapevolezza culturale." Annales, Series Historia et Sociologia 2017: (3) 27, 611-628.

\section{MIŠLENJA NASTAVNIKA O UČENJU U JEZIČNO I KULTURNO HETEROGENIM RAZREDIMA}

U radu su prikazani odabrani rezultati istraživanja o interkulturalnosti i interkulturalnom obrazovanju, koje je provedeno u šest regija u Sloveniji i Italiji u kojima su prisutne jezične i kulturne manjine. Istraživanje je ispitalo različite aspekte didaktike i obrazovanja učitelja i budućih učitelja, a također je obuhvatilo stavove učenika i njihovih roditelja o nastavi u jezično i kulturno heterogenim razredima. Rezultati predstavljeni u ovom radu mogu se podijeliti u sljedeće kategorije: (1) profesionalni, (2) osobni i kulturni razvoj nastavnika koji predaju u jezično i kulturno heterogenim razredima, (3) njihova 
percepcija nastave u ovim razredima općenito i posebno poučavanje jezika i (4) međuljudske odnose učenika. Rezultati ankete, u koju je bilo uključeno 281 učitelja, pokazuju da učitelji imaju izuzetno dobru percepciju vlastitog profesionalnog i kulturnog razvoja, ali pomalo nerado surađuju s drugim nastavnicima. lako većina učitelja vjeruje da istodobna prisutnost učenika različitih jezika i kultura ne ometa rad u učionici, ističu da poučavanje u jezično i kulturno heterogenim razredima zahtijeva dodatni rad od učitelja. Nastava ne utječe na poteškoće u komunikaciji među učenicima, baš kao što jezična raznolikost ne ometa razvoj komunikacijskih vještina u školskom jeziku podučavanja. Percepcija nastavnika o razvoju međuljudskih odnosa među učenicima pokazala se izuzetno pozitivnom.

Ključne riječi: jezično i kulturno heterogena nastava, mišljenja učitelja, didaktika, interkulturalno obrazovanje, talijanski jezik, slovenski jezik 